\author{
W. S. Feng, W. T. Yang and M. Y. Hsieh \\ Department of Electrical Engineering \\ National Taiwan University \\ Taipei, Taiwan, China
}

ABSTRACT

In this paper, we combine 1-D compaction with reshaping -a new action, to improve the result of compaction. Our algorithms change layout elements' shapes automatically according to some criteria and make neighboring layout elements' shapes as similar as possible. The more similar they are, the more compactable space we can obtain. The main part of our compaction is reshaping. If elements of certain $45^{\circ}$ layout can be reassigned their geometric shapes, we reshape them in order to gain more compactable space. Some experimental results of this $45^{\circ}$-compactor with and without reshaping of both manh
layouts are shown.

INTRODUCTION

Recently, computer-aided design for VLSI chip implementation has been investigated extensively. Compaction algorithm is of importance in the mask layout D compaction has no further impressive progress and problem [1]-[3]. With separate $X-$ and $Y$-compactions for 1-D compaction, the designer knows alt elements will be moved only along the direction of compaction. With two-dimensional compaction, however, the designer can no longer easliy predict the outcone because of the additional degree of freedom. So, it seens to be stuck in predicament for compaction desinger. Reshaping will be the turning point of layout compaction, we try to combine traditional one some amazing goal.

The most essential step for a compactor is to select a fit-for data structure favorable to trical features of layout masks, spatial data structures will be candidates for our choice. Comparisons between spatial data structures and our considerations will be described. Some spatial relationships between layout tiles will be defined for clarifying our algorithms. Then, we wil 1 discuss the one-dimensional moving strategy. After that, we will present the reshaping algorithm. Some experimental results and discussions will be summarized.

TRAPEZOIDAL CORNER STITCHING

This work was supported by the National Science Council, Taipei, Taiwan, under Grant NSC-81-0404-E-002-109 and -120 .
The varieties of the shapes of layout elements are wide and complicated, not like those rectangle layouts traditional compactions deal tangle layouts traditional compactions deal with. Using symbolic layouts will include a lo wil sy down cur slows mask layouts to me cooperated suitaple spatial data structure are the coltabiation for our arructure are the best combination for our conpaction. so, we use tration $[4]-[6]$.

1-D MOVING

The reshaping based on modified traditional 1-D compaction will lead to satisfying result. At compaction will lead to satisfying result. At ity of 2-D approaches. Our compaction contains two individual parts: reshaping and one-dimenional moving. We modify the scan-line algorithm ional moving. We modify the scan-line algorith into our 1-D moving algorlthm. Unlike original scan-line approach, $1-D$ moving doesn't need such a sophisticated computation for distance that is our $1-0$ moving doesn't chlef reason for traint our 1-D moving doesn e construct contraint graphs, and we fully take advantage of to data structure-trapezoldal corner stieching ing tiles instead of time-consuming computing.

The task of 1-D moving is to pick up an proper tile and get the distance it can move, then love it and all tiles associated with it to new locations. The problems for our 1-D moving aproach are as following:

- How to decide which tile to be chosen?

In there an easy way to get the information about neighborhoods around the tile chosen?

We will discuss solutions for these problems

I. Tile Sifter

The function of tile sifter is to choose a proper tile for 1-D moving. What is a proper tile?

A proper tile is a tile no un-moved tile blocks its way to move. All tiles left to a proper tile have been handled, hence, a proper tile is free to move left. Obviously, the direction to select a proper tile will be from left to right, just like the direction of a scan line movement. Because 1-D moving doesn't need such sophisticated processes, and it must match our data structure, we design a scan window for the se-
lection task. 


\section{Scan window}

Expanding the width of a scan line (it is zero) to non-zero gets a scan window. The width is set to the minimum width the technology allows. a movement unit of the scan window is the same -as its width, the minimum width. A scan window will be passed through all data planes at the same location. A scan line in constraint-graph approach gets the information of neighboring tiles, a scan window in our tile sifter extracts a proper tile for moving. Fig. I illustrates a scan window being passed through different layers.

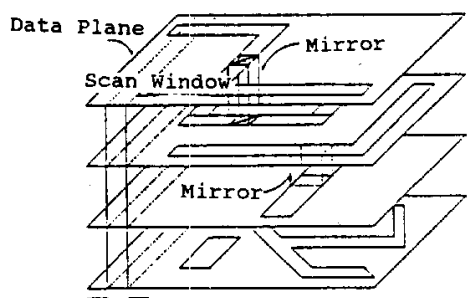

Min. width

Direction of Window Movement

Fig. 1 scan window

Area searching will be used to extract a proper Area searching will be used to extract a layers. tile within the scan window through all layers. on the same layer, area searching extracts tiles from left to right and from top to bottom. Every tile will be checked two qualities: solid tile or space tile; and whether auxiliary bit equals to 1 or not (an auxiliary) bit will be

3. Non-Horizontal Tile

The traditional method for doing 1-D compaction is to move layout elements and stretch horizontal elements instead of moving them. In $45^{\circ}$ mask layouts, tiles cannot be classified simply into two kinds: vertical and horizontal tiles, because shapes of tiles are full of variety. In addition to vertical and horizontal tiles,

Definition 4.1: Given tile $A, \operatorname{RUT}(A)$ ( or LUT(A)) is tile $B$ and RLT(A) (or LLT(A)) is tile $C$. Then tile $A$ is a non-horizontal tile, if:

$:(B \neq N U L L)$ and $\left(B_{-} 20 \leqq A_{-} 00\right),\left(A_{-10} \leqq B_{-30} 30\right)$

4. Design-Rule Window

The second problem we want to solve is moveable distance for the tile chosen by tile sifter described above. In fact, not only a tile will be moved, all tiles connected to that tile chosen by tile sifter will, too. It is unnecessary to use sophisticated computation ta get moveable distance, simple one is enough for $1-D$ moving.

For a tile on some layer (e.g. poly layer), there are several constraints between this tile and other tiles on different layers (e.g. poly poly, poly-n diffusion and poly-p diffusion). design-rule window is created for specific design rule constraint, there are different design rule windows for different constraints in different layers.

\section{Creation of Design-Rule window}

A design-rule window is not like a scan window, it doesn't move. The right edge of a designrule window is extended left edge of a tile. the length of extra portion depends on the concerned constraint. The width of a design-rule window also depends on the concerned constraint. As shown in Fig. 2, a poly tile owns two designrule windows with different sizes on different layers such as poly-n diffusion.

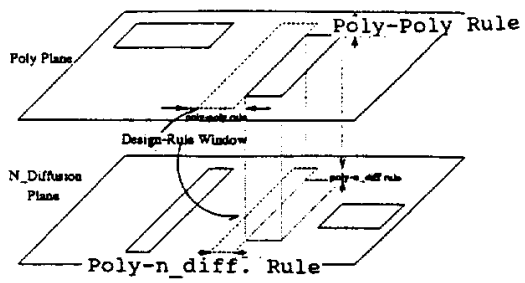

Fig. 2 Design-rule window

6. Function of Design-Rule window

Because design-rule windows are created under design rules, there must be no solid tile found within windows. If it does, it violates the design rules in original layout. our compactor informs users of that fact and stops proceeding. otherwise, area searching will extract all space tiles in windows of all relative layers, and the minimum value among all minimum widths of space tiles will be the moveable distance for this tile which the design-rule window belongs to. A minimum width of a tile is the smaller length between top edge and bottom edge of it. Fig. 3 shows the moveable distance for tile on a single layer.

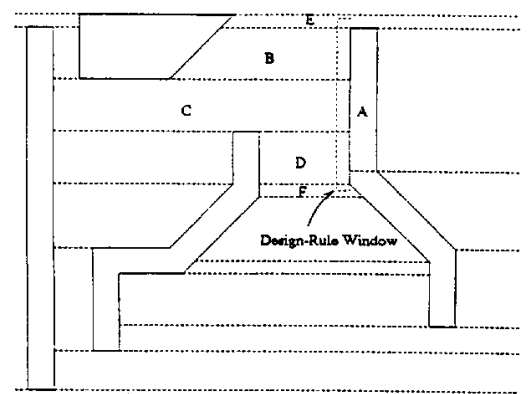

Fig. 3 Moveable distance of $\mathrm{A}$ (in a layer) $=$ min(width(B), width(C), width(D)

Because the feature of corner stitching - no space tile will be next immediately to a space tile, a space tile must lies between solid tiles. All space tiles found in a design-rule window created by one solid tile, whose left neighbor tiles are other solid tiles, that is why the minimum width of all space tiles lying within design-rule windows is the moveable distance for a concerned tile. 


\section{Overall Moveable Distance}

All tiles connected to the tile chosen by tile sifter must be taken into account for overall
moveable distance, because these tiles will be disconnected to it at new location after moving that chosen tile. There are four kinds of such connected tiles:

- connection tiles on the same layer,

- connection through contacts, and

- connection through Moses.

\section{RESHAPING}

Reshaping is one of novel ideas used in our compactor. What is reshaping? In brief, reshaping gives one layout element a new shape and changes its shape into more compactable one. It is shown in Fig. 4. Without doubt, after such rearrangement we can compact entire chip into much denser size than before. Problems face with us are:

- Reshaping will affect tiles' locations, so not all tiles can be reshaped. How to decide whether one tile is reshapeable or not is an essential problem.

- What is the optimal new shape which a tile shoula be reshaped? What plied to reshape?
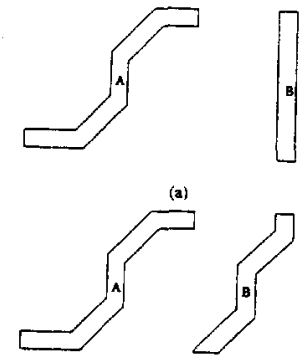

(b)

Fig. 4 Basic idea of reshaping, (a) before reshaping and (b) after reshaping.

As usual, our ideas are presented below.

1. Conditions to Reshape

we conclude the following conditions. If a tile meets these conditions then it is not reshapeable. If we concern tile $A$, these conditions are

- The shape of $A$ is not S1, S5 or S9. Because, reshaping does no special good for tiles to tile A according to the reshaping method we use, it will be described,later.

- Connection tiles through contacts or MOSFETs

will be not connected to the tile after rewill be not connected to the tile after reshape tile A. This is obvious, disce

- will make circtits ma same layer cannot connection tiles on then reshape tile $A$.

2. New Shape Determining

Determining a new shape is classified into two aspects: local reshaping and bending reshaping. A local reshaping changes a tile's shape slight ly and a bending reshaping bends a tile into tiles.

a. Local Reshaping
Local reshaping is some kind of "divide and conquer" strategy, and it cuts a layout element into several smaller tiles and reshapes them. Local reshaping wants to change least and reaches the expected goal of reshaping. The first step of local reshaping is to cut a tile intc some single-neighbor tiles. A single-neighbor tile is a tile which owns one and only one left neighbor tile and right neighbor tile.

b. Single-Neighbor Tile

We take advantage of the feature of corner stitching to cut through a tile into several single-neighbor tiles. Just delete all space tiles extracted from a tile's left neighborhood, it will cut that tile into single-neighbor tiles automatically. Fig. 5 shows a result of deleting space tiles left to one tile.

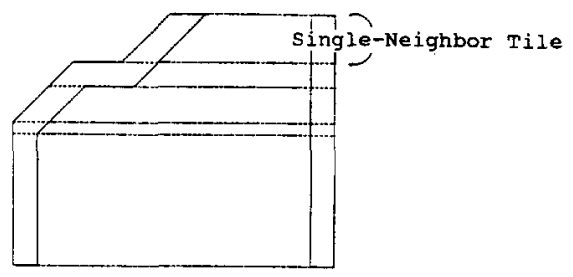

Fig. 5 Cut into single-neighbor tiles.

c. Best Shape to Reshape

For two neighbor solid tiles (e.g. tile $A$ and tile $B$ in Fig. 6(a), the best situation for compaction is the right edge of tile $A$ and left of $B$ have the same declination angles, like tile $B$ in Fig $6(\mathrm{~b})$. of course, the right edge of tile in must tollow declination of the left edge tile tiles right to tile $\mathrm{can}$ be tries right to tile $B$ can be moved left Lurther. easily.

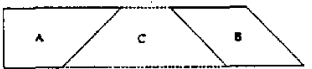

(a)

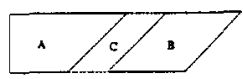

(b)

Fig. 6 Best shape to reshape, (a) before reshaping and (b) after reshaping.

If top equals to bottom then it is unnecessary to change the shape, the original shape is good enough for compaction. BL(ti) is to extract the space tile immediately right to $t i$, and to compute the length of top and bottom edge of the space tile, LENGTH(ti). By top and bottom, we can easily get the same declination angles described above. Because the same declination angles result in that top and bottom of the
space tile must be equal.

d. Bending Reshape 
In some cases, an unreshapeable tile will waste a remarkable uncompactable space. In Fig. 7 , tile $C$ is unreshapeable with tile $B$, it leaves and $c$.

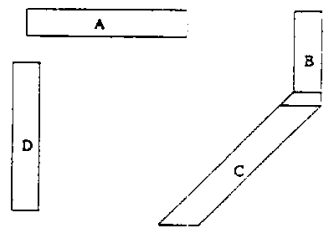

Fig. 7 An example for an unreshapeable tile.

Bending reshape is performed on such a situation, it replaces a tile with two tiles - one vertical and one horizontal. In other words, it bends a tile. Fig, 8 shows bending reshape on Fig 7. The space between tile $C$ and $D$ as shown in 7 is 7 compactable as given in Fig. 8 That is bending reshape.

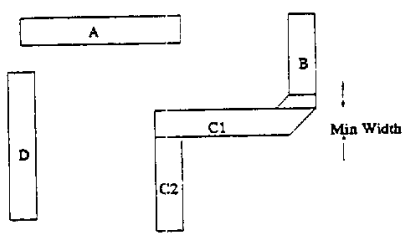

Fig. 8 Tile $\mathrm{C}$ is compactable after bending reshape.

e. Conditions to Bending Reshape

The conditions which must be checked before bending reshape on a tile are as follows:

- The tile is unreshapeable for local reshaping.

- The shape of the tile is 55 or $\$ 9$.

- Connection tiles through contacts or MOSFETs are still connected to the tile after reshaping tile $A$.

- The height of a tile is no less than double minimum width the technology permits.

We only concern two types of shapes that often cause uncompactable space described above, that is 55 and 59 . In the same time, we don't need to check those connection tiles on the same layer, because the ends of the tile to be reshaped will not be changed. It is important to assure that the height must be no less than double minimum width, otherwise, it will violate the design rules after replacing it with two tiles.

f. Methods of Bending

simply, we delete an old tile then insert two in Fig. 8 .

\section{EXPERIMENTAL RESULT}

We design several simple layouts to test our algorithms. We test in two phases: one does reshaping and one doesn't. Detailed results on this work are described in Table

Reshaping is optional. operation, if user needs faster result or $45^{\circ}$ masks are few, it can rule out the reshaping part-just do $1-D$ moving part.
Of course, our compaction is not perfect. Regular layouts limit the function of reshaping, that is because the shapes of masks in regular layout are very similar. Since the critical situation is manhattan layout, all tiles are shape S1 in manhattan layout. So, it is unreshapeable according to our algorithm. By our observations. non-manhattan layouts are likely to be reshaped into manhattan layouts. The chief reason is the contours of all chips are rectangle.

Table 1. 1-D Moving and Reshaping Result

\begin{tabular}{|c|c|c|ccc|ccc|}
\hline Exampie & \#Tiles & $S_{b}$ & \multicolumn{3}{|c|}{ no reshaping } & \multicolumn{3}{|c|}{ reshaping } \\
\cline { 4 - 9 } & & & $N_{m}$ & $S_{a}$ & CPU [secs] & $N_{m}$ & $S_{a}$ & CPU [secs] \\
\hline S0127 & 32 & 1538 & 20 & 1041 & 17.33 & 23 & 665 & 32.35 \\
\hline 53061 & 49 & 2095 & 47 & 917 & 30.59 & 23 & 715 & 35.44 \\
\hline 53063 & 40 & 1551 & 21 & 1139 & 10.81 & 11 & 1132 & 8.81 \\
\hline S3181 & 50 & 1826 & 29 & 1624 & 12.88 & 23 & 931 & 17.00 \\
\hline S3182 & 46 & 1921 & 10 & 1777 & 6.57 & 12 & 1633 & 11.80 \\
\hline S3103 & 89 & 5172 & 51 & 2481 & 25.74 & 46 & 2422 & 37.92 \\
\hline S0602 & 85 & 31178 & 132 & 19969 & 220.87 & 196 & 10165 & 361.82 \\
\hline
\end{tabular}

\#Tiles : the number of tiles within an example.

$S_{\mathrm{b}}$ : space area before compacting $\left[\lambda^{2}\right]$.

$S_{a}$ : space area after compacting $\left[\lambda^{2}\right]$.

$N_{m}$ : the number of moved tiles.

CONCLUSIONS

In practical applications, non-manhattan layout is more useful and preferable than manhattan layout. In addition to its flexibility, it produces denser layout area than manhattan layout. In nowadays manufacture technology, $45^{\circ}$ masks are available. So, compactors which can deal with $45^{\circ}$ layouts are necessary. In this work, we present a new $1-D$ compactor and it can accept both manhattan and $45^{\circ}$ mask layouts. At the same time, $45^{\circ}$ masks provide a great chance the same time, 45 masks provide a great chance portant feature of our compaction-reshaping.

Reshaping really improve the results of compaction in our experiments, but it is limited py some conditions. And our criteria of reshapeability-check are not sophisticated enough to handle all possible not sophisticated enough to handle all possible cases, there are a lot of cases we can not deal with and that degrade the power of reshaping. Nevertheless, reshaping should be an easier approach to compaction design. It doesn't need involve the complicated
situations of 2-D compaction, but obtains more denser area than traditional compaction does.

REFERENCES

1. W. Wolf, Proc. 22nd Design Automation Conf., (1985), pp. 90-91.

2. H. Shin, A. Sangiovanni-Vincentelli, and $C$. Sequin, Proc. 23nd Design Automation Conf. (1986), pp.115-122.

3. Johannes Waterkamp, Rainer wicke and Rainer Bruck, Proc. 26th Design Automation Conf. (1989), pp. 296-301.

4. D. Marple, M. Smulders and H. Hegen, IEEE Trans. on CAD, vol. 9 , No l (1990) pp.66-87.

5. 3. K. Ousterhout, IEEE Trans. on CAD of Circuits and systems, (1984) pp.87-100.

6. D. Marple, M. Smulders, and H. Hegen, Proc. 25 th Design Automation conf., (1988) pp.396402 . 